\title{
Quantités ingérées par des agneaux en allaitement maternel pendant la première semaine de vie
}

\author{
Yseult VILLETTE $\left({ }^{*}\right)$ et M. THERIEZ \\ avec la collaboration de J.P. BRuN \\ I.N.R.A., C.R.Z.V. de Theix, Laboratoire de la Production ovine \\ F 63122 Ceyrat
}

\begin{abstract}
Résumé
Les quantités de lait ingéré ont été mesurées par l'utilisation d'un marqueur de l'eau sur 67 agneaux âgés de 2 à 5 jours, de génotype Limousin $(\mathrm{L})$, Romanov $\times$ Limousin $(\mathrm{RL})$, He-de-France $(\mathrm{IDF})$ et IDF $\times \mathrm{RL}(\mathrm{IRL})$. Rapportées au poids métaboliquc, les quantités d'eau bue sont voisines entre génotypes et sont indépendantes de la taille de la portée allaitée ou du poids à la naissance des agneaux. En portée double, la production laitière des brebis en première semaine, dépend de la seule demande effectuce par les agneaux.

Le besoin d'entretien estimé à partir de la régression de la vitesse de croissance $\left(\mathrm{kg} / \mathrm{kg} \mathbf{P}^{(1,75}\right)$ sur les quantités d'eau bues $\left(\mathrm{kg} / \mathrm{kg} \mathbf{P}^{\left(0, r^{\circ}\right)}\right)$ est plus ćlevé en génotype IDF

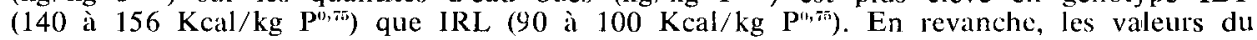
rendement de l'énergie métabolisable sont voisines entre elles $(0,43$ à 0,51$)$ mais inférieures à celles observées dans la bibliographie.
\end{abstract}

\section{Introduction}

En début de vie postnatale, l'acquisition de l'immunité passive dépend d'une ingestion précoce de colostrum. Les quantités de colostrum puis de lait ingérées conditionnent également la résistance de l'agneau au froid et le démarrage de la croissance du jeune animal. Mesurées sur des agneaux en allaitement artificiel nourris à volonté, les consommations au cours de la première semaine de vie sont voisines de $68 \mathrm{~g} \mathrm{MS} / \mathrm{kg}$ P0,75 (VilletTe \& Theriez, 1981). Il est cependant nécessaire de préciser ces données en allaitement maternel et de déterminer si, dès la première semaine de vie, la taille de la portée allaitée peut être un facteur limitant des quantités ingérées. Les méthodes fondées soit sur la pesée avant et après tétée, soit sur la traite après injection d'ocytocine, ne peuvent être appliquées car elles nécessitent des périodes de séparation entre

(") Adresse actuelle : Laboratoire de Recherches de la Chaire de Zootechnie (I.N.R.A.) de l'Institut national agronomique Paris-Grignon, 16, rue Claude-Bernard, 75231 Paris Cedex 05 (France). 
la mère et ses agneaux beaucoup plus longues que l'intervalle naturel entre tétées (15 à 25 tétées en 12 heures de jour, EwBank, 1967). Le développement de la méthode de mesure des quantités d'eau bue par injection d'un marqueur de l'eau permet d'estimer la quantité de lait bu sans perturber les comportements de tétée. Nous avons appliqué cette méthode à 46 brebis allaitant 67 agneaux au cours de leur première semaine de vie. Les données obtenues nous ont permis d'estimer les besoins d'entretien et le rendement de l'énergie métabolisable (EM) pour la croissance (kpf).

\section{Matériel et méthodes}

Les mesures ont été effectuées au cours d'un agnelage de printemps sur 24 brebis Ile-de-France (IDF) allaitant des agneaux de même race et, pour l'agnelage d'automne de la même année, sur 10 brebis Limousines (agneaux Limousin et Romanov $\times \mathrm{Li}$ mousin, symbole commun : L) et 9 brebis Romanov $\times$ Limousin allaitant des agneaux croisés IDF (IRL). La répartition des agneaux en fonction de la taille de la portée et du sexe est présentée dans le tableau 1. Les agneaux ont reçu à l'âge de 1 à 2 jours une injection intraveineuse d'eau deutériée à raison de 1 p. 1000 du poids vif.

\section{Tableau 1}

Répartition des agneanx en fonction du génotype, de la taille de la portée allaitée et du sexe.

Distribution of lambs within genotype, suckling litter size and sex.

\begin{tabular}{|c|c|c|c|}
\hline & \multicolumn{3}{|c|}{ Genotype maternel - Maternal genotype } \\
\hline & $\begin{array}{l}\text { Limousine } \\
\quad(\mathrm{L})\end{array}$ & $\begin{array}{c}\text { Romanov } \\
\times \\
\text { Limousine } \\
\text { (RL) }\end{array}$ & $\begin{array}{l}\text { Ile-de-France } \\
\text { (IDF) }\end{array}$ \\
\hline $\begin{array}{l}\text { Génotype des agneaux } \\
\text { Lamb genotype }\end{array}$ & $\mathrm{L}, \mathrm{RL}^{*}$ & IRL & IDF \\
\hline $\begin{array}{l}\text { Tous agneaux } \ldots \ldots \ldots \ldots \\
\text { All lambs }\end{array}$ & 15 & 18 & 34 \\
\hline $\begin{array}{l}\text { Alïaités simples } \ldots \ldots \ldots \ldots \ldots \\
\text { Single suckling }\end{array}$ & 5 & 0 & 14 \\
\hline $\begin{array}{l}\text { Allaités doubles } \\
\text { Twin suckling }\end{array}$ & 10 & 18 & 20 \\
\hline $\begin{array}{l}\text { Mâles } \ldots \ldots \ldots \ldots \ldots \ldots \ldots \\
\text { Males }\end{array}$ & 7 & 7 & 17 \\
\hline $\begin{array}{l}\text { Femelles } \\
\text { Females }\end{array}$ & 8 & 11 & 17 \\
\hline
\end{tabular}

(*) Symbole commun dans la suite des tableaux : $\mathbf{L}$. Common symbol in the other tables : $L$. 
Les 3 jours suivants, environ à la même heure notée exactement, les agneaux ont été pesés puis $5 \mathrm{ml}$ de sang ont été prélevés à l'aide d'une seringue héparinée et conservés à $-18{ }^{\circ} \mathrm{C}$ jusqu'au traitement. La teneur en eau deutériée de l'eau du sang a été déterminée par spectrophotométrie infrarouge selon la méthode de Tissier et al. (1978). Les quantités d'eau bue ont alors été calculées à partir de la pente d'élimination (a) du marqueur selon l'équation suivante (BocQuiER, données non publiées) obtenue sur des animaux d'âge voisin et maintenus dans des conditions d'environnement similaires.

$$
\text { Eau bue }=10^{4} \times 229,33 \mathrm{a}+13,57 \quad \mathrm{R}=0,926 \quad \mathrm{n}=24
$$

a : pente d'élimination du marqueur exprimée en logarithme décimal de la concentration en eau deutériée par minute.

Cette méthode suppose que le volume d'eau corporel reste constant pendant la période de mesure ce qui n'est pas le cas pour des animaux en croissance. Selon les résultats de Dove \& FREer (1979), la sous-estimation qui en résulte est faible (moins de 3 p. 100) lorsque les mesures sont limitées à 3 jours.

A partir des quantités d'eau bue, nous avons estimé les quantités de lait, d'énergie brute (EB), digestible (ED) et métabolisable (EM) ingérées en fonction de résultats disponibles dans la bibliographie. Les valeurs retenues sont les suivantes : teneur en matière sèche du lait : 22 p. 100 (Perrin, 1958; Gardner, Hogue \& Bensadoun, 1964 ; Peart, 1972 ; Sans Arias, Ovejero \& Zorita, 1973) ; digestibilité de l'énergie : $0,922+0,0095 \times$ poids à la naissance (Houssin \& DAvicco, 1979), EM/ED : 0,96 (WAlKer \& Norton, 1971). Compte tenu de la variabilité des données disponibles, deux hypothèses ont été retenues pour la teneur en énergie brute du lait : 1400 et $1550 \mathrm{kcal} / \mathrm{kg}$ (Perrin, 1958 ; Gardner, Hogue \& Bensadoun, 1964 ; Peart, 1972 ; Sans ARIAS, OVEJERo \& Zorita, 1973).

\section{Résultats}

\section{A. Quantités ingérées et croissance des agneaux}

Les quantités journalières de lait ingéré (22 p. $100 \mathrm{MS})$ entre le $3^{\circ}$ et le $5^{\bullet}$ jour de vie ont varié de moins de $768 \mathrm{~g}$ à plus de $1664 \mathrm{~g}$ avec une valeur moyenne de $1180 \mathrm{~g} \pm 280 \mathrm{~g}$. Pendant la première semaine de vie, la croissance a été en moyenne de $259 \pm 86 \mathrm{~g} / \mathrm{j}$.

\section{Influence du poids à la naissance et de la taille de la portée}

Les consommations $(\mathrm{g} / \mathrm{agneau} / \mathrm{j})$ sont d'autant plus élevées que le poids à la naissance augmente ou que la taille de la portée diminue (tabl. 2 et 3 ). Poids à la naissance et taille de la portée sont deux facteurs liés et l'analyse de covariance indique qu'étudiée à même taille de portée, l'influence du poids à la naissance persiste. En revanche à même poids à la naissance, l'influence de la taille de la portée n'est plus significative. Toutes tailles de portées confondues, une augmentation de $1 \mathrm{~kg}$ à la naissance accroît les consommations journalières de $276 \mathrm{~g}$ de lait $(22 \mathrm{p} .100 \mathrm{MS}$ ). Cependant, rapportées au poids métabolique $\left(\mathrm{P}^{0,75}\right)$ moyen des $3^{\circ}$ au $5^{\circ}$ jours, les 
quantités ingérées sont indépendantes du poids initial (tabl. 2). L'indice de consommation en moyenne égal à $4,6 \pm 1,2 \mathrm{~kg}$ de lait $(22 \mathrm{p} .100 \mathrm{MS}) / \mathrm{kg}$ de gain de poids vif est indépendant du poids à la naissance (tabl. 2). L'augmentation de la vitesse de croissance avec le poids initial (tabl. 2) est donc due aux seuls effets de ce poids sur les quantités ingérées.

Tableau 2

Influence du poids à la naissance $(P N)$ sur les consommations journalières et la croissance pendant la première semaine de vie.

Influence of birth weight ( $P N)$ on daily milk intake and weight gain of lambs during the first week.

\begin{tabular}{|c|c|c|c|c|}
\hline & $\mathrm{y}=\mathrm{a}+\mathrm{bPN}(\mathrm{kg})$ & $\sigma \mathrm{b}^{*}$ & $\mathrm{R}^{*}$ & $\mathbf{P}$ \\
\hline \multicolumn{5}{|l|}{$\begin{array}{l}\text { Quantités de lait bu ( } 22 \% \text { MS) } \\
\text { Milk intake }(22 \% \text { DM) }\end{array}$} \\
\hline$-\mathrm{g} / \mathrm{j}, \ldots \cdots \cdots \cdots \cdots \cdots$ & $295+216 \mathrm{PN}$ & 29 & $+0,69$ & 0,001 \\
\hline 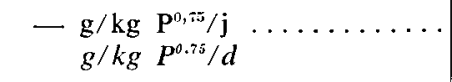 & $341+7 \mathrm{PN}$ & 9 & $+0,11$ & NS \\
\hline \multicolumn{5}{|l|}{$\begin{array}{l}\text { Croissance } 0-7 \mathrm{j} \\
\text { Weight gain } 0-7 \text { days }\end{array}$} \\
\hline$-\mathrm{g} / \mathrm{j} \ldots \ldots \cdots \cdots$ & $74+46 \mathrm{PN}$ & 11 & $+0,47$ & 0,01 \\
\hline \multicolumn{5}{|l|}{$\begin{array}{l}\text { Indice de consommation } \\
\text { Feed conversion ratio }\end{array}$} \\
\hline $\begin{array}{l}\text { - } \mathrm{kg} \text { lait } / \mathrm{kg} \text { gain de poids vif } \\
\mathrm{kg} \text { milk/kg live weight }\end{array}$ & $4,0+0,2 \mathrm{PN}$ & 0,2 & $+0,12$ & NS \\
\hline \multicolumn{5}{|c|}{$\begin{array}{l}\left.{ }^{*}\right) \\
\text { ob : Ecart-type du coefficient de régression. } \\
\text { Standard deviation of the regression coefficient. }\end{array}$} \\
\hline 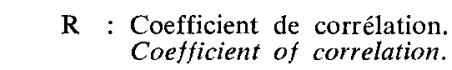 & & & & \\
\hline
\end{tabular}

\section{Influence du génotype et du sexe}

Ajustées par analyse de covariance pour un poids moyen à la naissance de $3,8 \mathrm{~kg}$, les consommations journalières de lait ( 22 p. $100 \mathrm{MS})$ augmentent des génotypes IDF à $I R L$ et $L$ ou des agneaux mâles à femelles mais les différences ne sont pas significatives (tab1. 4). Ces deux facteurs sont également sans effet significatif sur la vitesse de croissance ou l'indice de consommation. 


\section{TABLEAU 3}

Influence du mode d'allaitement sur les consommations journalières de lait et la croissance pendant la première semaine de vie.

Influence of suckling litter size on the daily milk intake and the live weight gain of lambs during the first week.

\begin{tabular}{|c|c|c|}
\hline & \multicolumn{2}{|c|}{ Mode d'allaitement - Suckling litter size } \\
\hline & Simple - Single & Double - Twin \\
\hline $\begin{array}{l}\text { Poids à la naissance }(g) \ldots \ldots \ldots \ldots \ldots \ldots \\
\text { Birth weight }(g)\end{array}$ & $4,4 \pm 0,6^{\Lambda}$ & $3,6 \pm 0,6^{B}$ \\
\hline $\begin{array}{l}\text { Quantités de lait bu ( } 22 \% \text { MS }) \\
\text { Milk intake }(22 \% D M)\end{array}$ & & \\
\hline$-\underset{g / d}{\mathrm{~g} / \mathrm{j}} \cdots \cdots \cdots \cdots \cdots \cdots$ & $1327 \pm 300^{a}$ & $1113 \pm 244^{b}$ \\
\hline $\begin{aligned}-\mathrm{g} / \mathrm{kg} P^{0,75} / \mathrm{j} & \ldots \\
& g / k g P^{0,7 s} / d\end{aligned}$ & $376 \pm 70^{\mathrm{a}}$ & $375 \pm 51^{a}$ \\
\hline $\begin{array}{l}\text { Crojssance } 0-7 \text { jours } \\
\text { Weight gain } 0-7 \text { days }\end{array}$ & \multirow[b]{2}{*}{$297 \pm 112^{a}$} & \multirow[b]{2}{*}{$241 \pm 60^{\mathrm{a}}$} \\
\hline$-\underset{g / d}{g / j} \cdots \cdots \cdots \cdots$ & & \\
\hline $\begin{array}{l}\text { Indice de consommation } \\
\text { Feed conversion ratio }\end{array}$ & \multirow[b]{2}{*}{$5,0 \pm 1,50^{\mathrm{a}}$} & \multirow[b]{2}{*}{$4,8 \pm 1,0^{\mathrm{a}}$} \\
\hline $\begin{array}{l}\text { - } \mathrm{kg} \text { lait } / \mathrm{kg} \text { gain de poids vif } \\
\mathrm{kg} \text { milk/g live weight gain }\end{array}$ & & \\
\hline
\end{tabular}

a, b ; A, B : Dans les lignes, Ies données n'ayant pas de lettres communes sont significativement différentes au seuil de 5 p. $100(\mathrm{a}, \mathrm{b})$ et 1 p. $100(\mathrm{~A}, \mathrm{~B})$.

Within lines, results with no common letters are significantly different at the $5 \mathrm{p} .100$ $(a, b)$ or 1 p. $100(A, B)$ levels.

B. Estimation $d u$ besoin d'entretien et du rendement de l'énergie pour la croissance $(k p f)$

Le besoin énergétique d'entretien ainsi que la valeur du kpf ont été estimés par régression linéaire du gain de poids vif $\left(\mathrm{kg} / \mathrm{kg} \mathrm{P}^{0,75}\right)$ sur les quantités d'eau bue $\left(\mathrm{kg} / \mathrm{kg} \mathrm{P} \mathrm{P}^{0,75}\right.$ ) (fig. 1). Tous génotypes confondus, le besoin d'entretien s'élève à 110 ou $122 \mathrm{kcal}$ E.M. $/ \mathrm{kg} \mathbf{P}^{0,75}$ (tabl. 5) et la valeur du $\mathrm{kpf}$ à 0,49 ou 0,44 selon la valeur retenue pour la teneur en énergie brute du lait (1 400 ou $1550 \mathrm{kcal} \mathrm{EB} / \mathrm{kg}$ respectivement).

Estimées séparément pour les agneaux IDF et IRL, les valeurs du kpf sont assez voisines (tabl. 5). En revanche, le besoin d'entretien des animaux IDF (140 ou $156 \mathrm{kcal}$ E.M./kg $\mathrm{P}^{0,75}$ ) est nettement supérieur à celui des animaux IRL (92 ou $102 \mathrm{kcal}$ E.M./kg $\mathrm{P}^{0,75}$ ). 


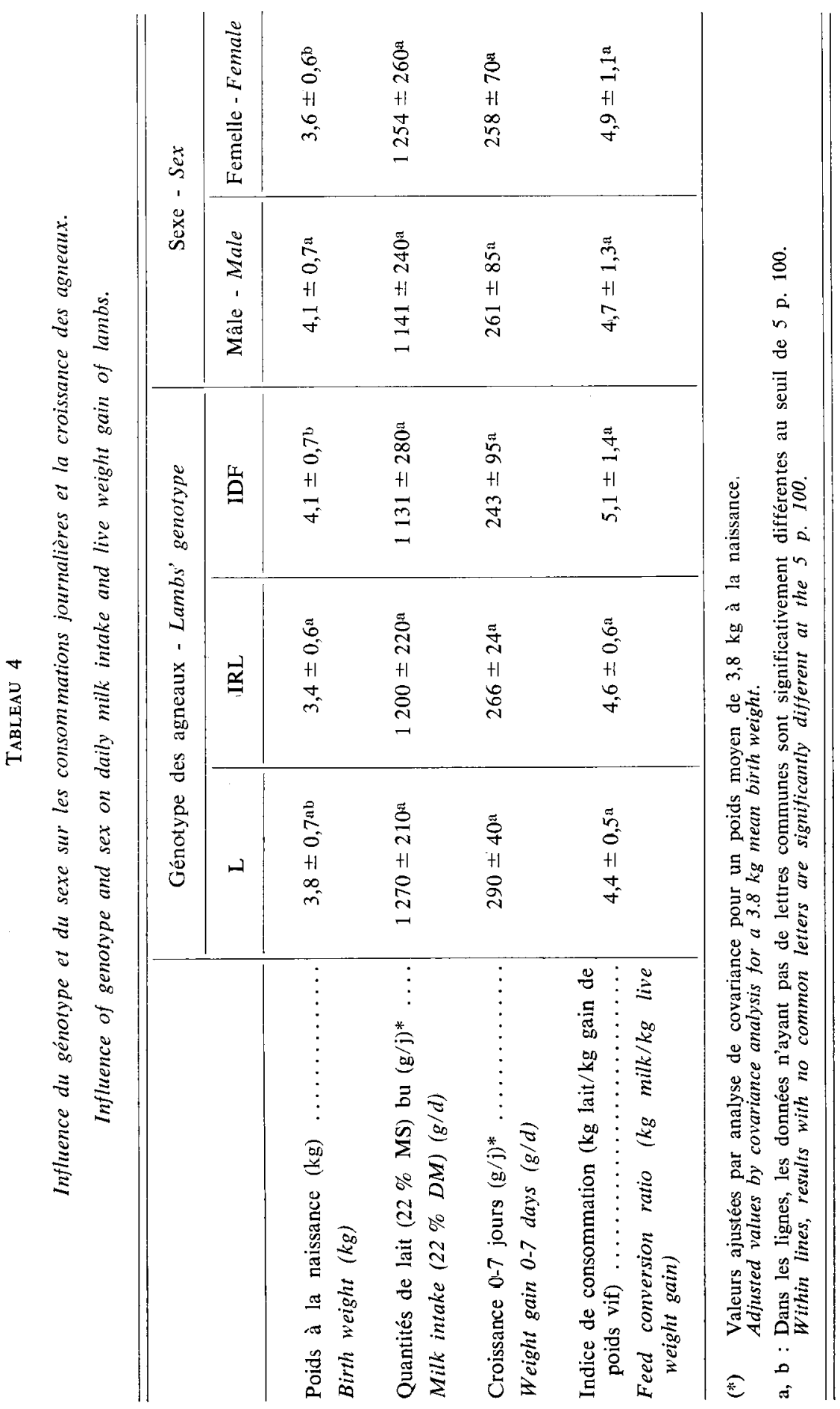




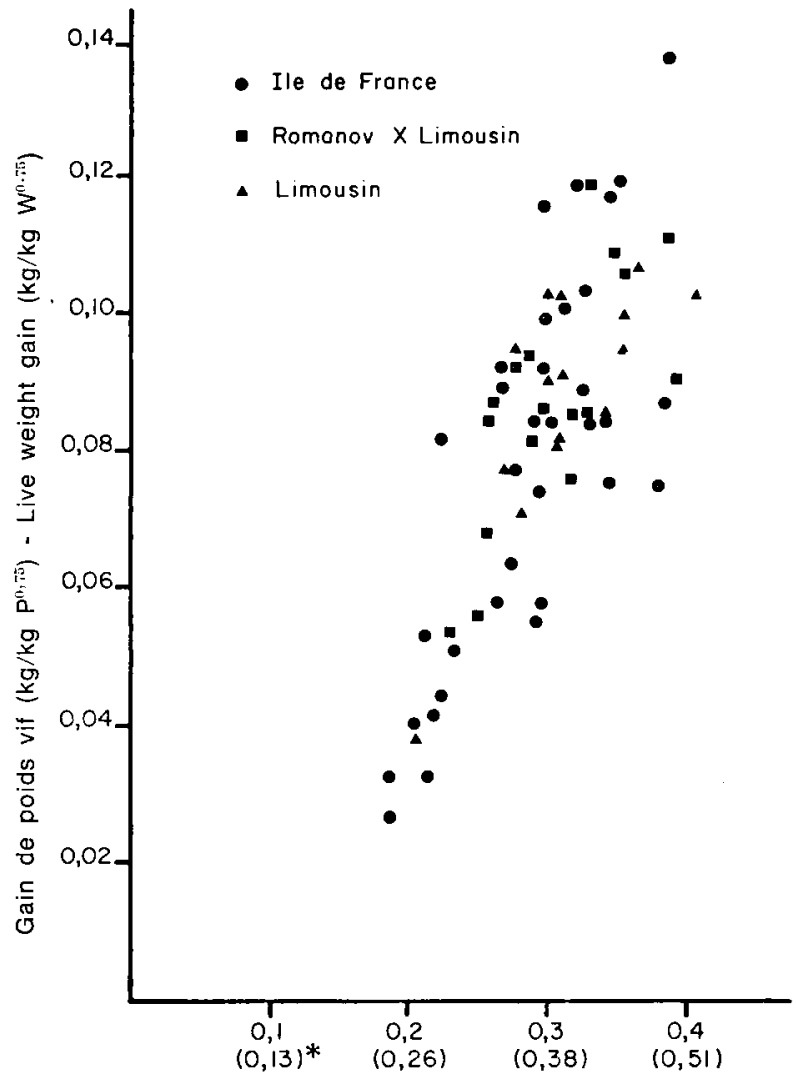

Eau bue $\left(\mathrm{kg} / \mathrm{kg} P^{0.75}\right)$ - Water intake $\left(\mathrm{kg} / \mathrm{kg} \mathrm{W}^{0.75}\right)$

FIG. 1

Relation entre le gain de poids vif pendant la première semaine de vie et les quantités d'eau bues.

Relationship between the live weight gain during the first week and the water intake.

* ( ) : Quantités correspondantes de lait bues (22 p. 100 de M.S.).

Corresponding milk intake (22 p. 100 of D.M.). 


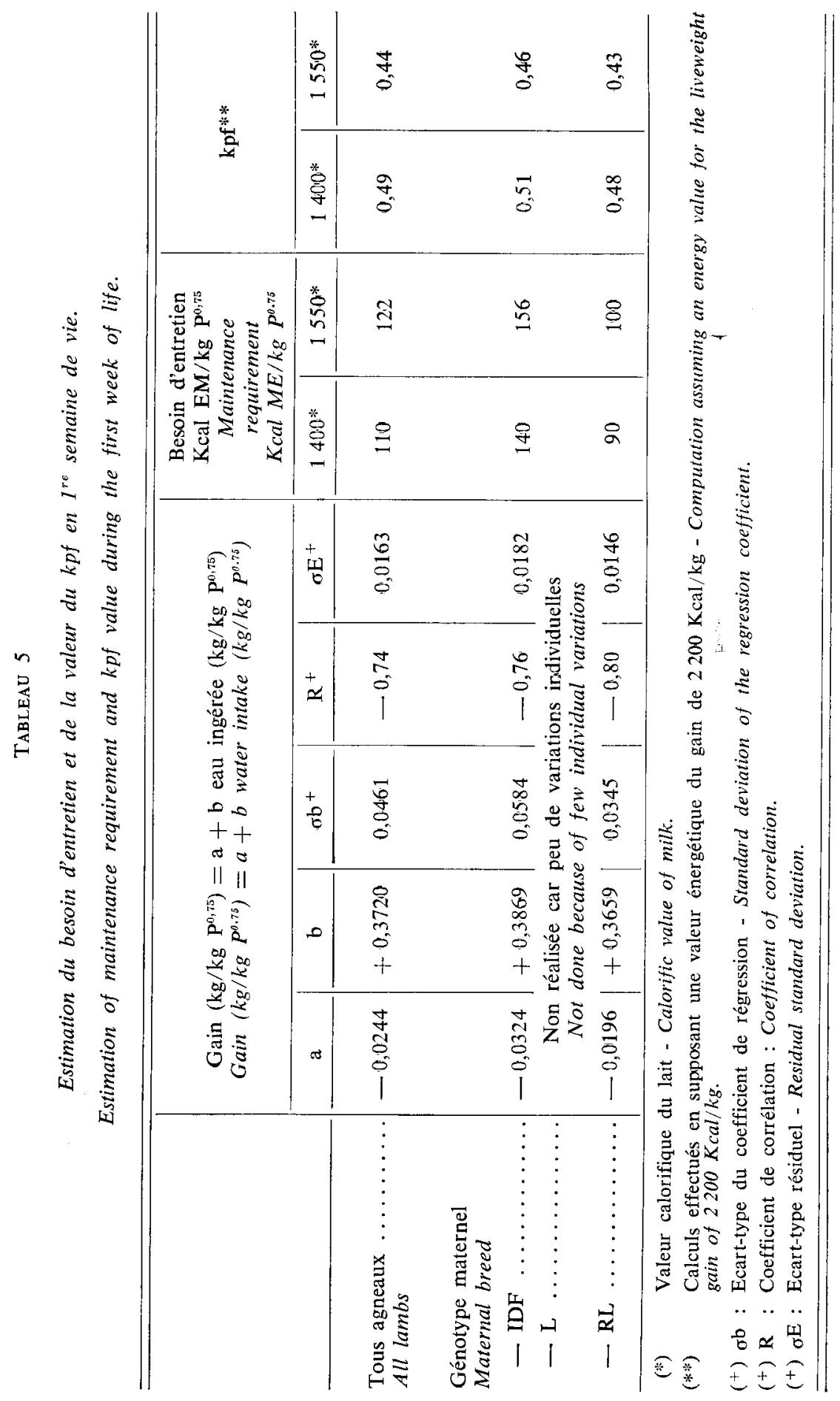




\section{Production laitière des brebis}

Les productions laitières des brebis allaitant 2 agneaux sont en moyenne de $2,3 \pm 0,3,2,0 \pm 0,4$ et $2,0 \pm 0,4 \mathrm{~kg}$ de lait (22 p. $100 \mathrm{MS}$ ) par jour respectivement pour les génotypes maternels $\mathrm{L}$, RL et IDF. Ces valeurs représentent $173 \mathrm{p}$. 100 et 163 p. 100 des valeurs obtenues en portée simple respectivement pour les brebis $\mathrm{L}$ et IDF. En portée double, la production laitière des brebis augmente avec le poids de la portée (fig. 2), de façon linéaire en race maternelle RL, curvilinéaire en race IDF où la production maximale est de 2,2 à $2,4 \mathrm{~kg}$ de lait par jour. Cependant nos données sont en nombre trop limité pour déterminer si ces résultats reflètent des différences raciales de potentiel laitier.

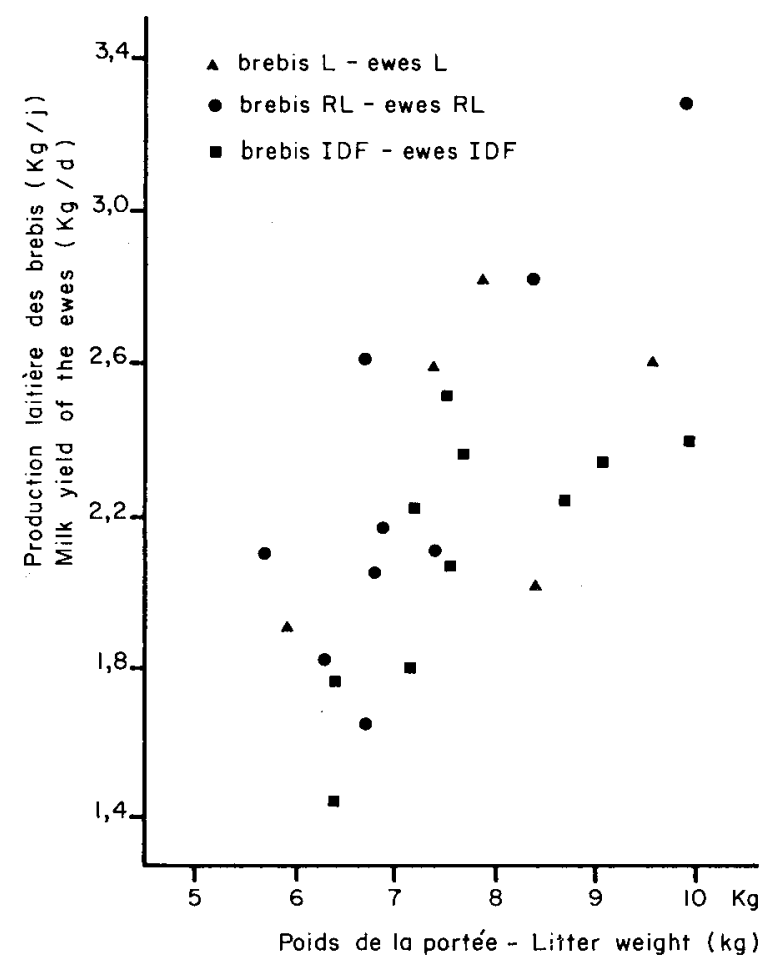

FIG. 2

Relation entre le poids de la portée

et la production laitiere des brebis allaitant deux agneaux.

Relationship between litter weight and milk yield of twin-suckled ewes.

Deux équations d'estimation de la production laitière (Y) à partir de la croissance de la portée ont été calculées (tabl. 6). L'équation (1), fonction du poids métabolique $\left(P^{0,75}\right)$ et du gain de poids vif $(G)$ tient compte de la répartition des nutriments entre l'entretien et la croissance. L'équation (2) est fonction du poids $\mathbf{P}$ et du gain de poids vif élevé au carré $\left(\mathrm{G}^{2}\right)$ selon le modèle (3) proposé par Robinson, Foster \& Forbes (1969). 


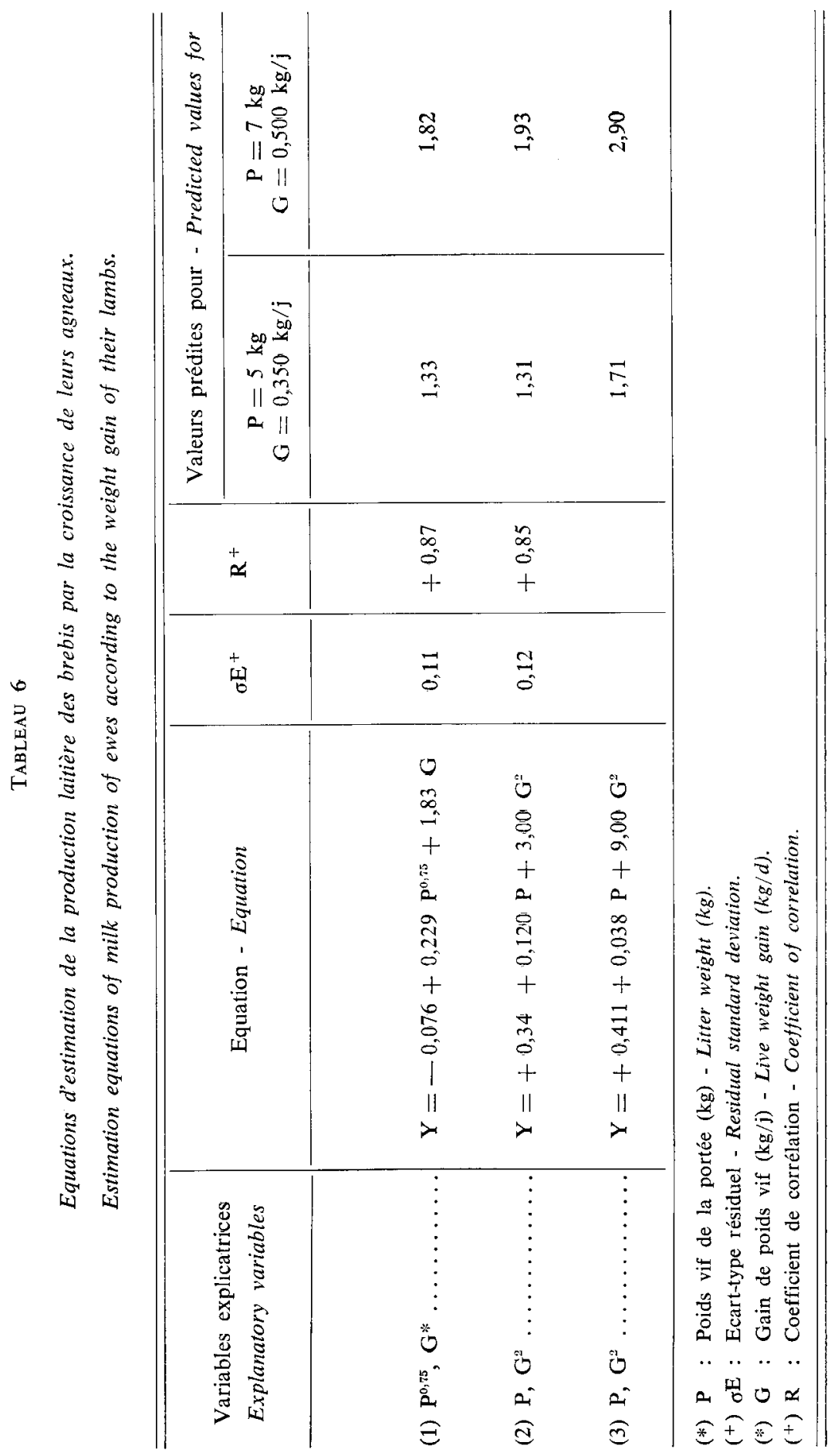


L'équation (2) donne une estimation légèrement moins bonne que la première avec des coefficients de régression nettement différents de ceux proposés par RoBinson, Foster \& Forbes (1969) pour des agneaux d'âge plus élevé. Les productions laitières estimées pour deux exemples de poids et de croît de portée (tabl. 6) sont voisines à partir des équations (1) et (2) mais surévaluées de 400 à $1000 \mathrm{~g}$ si on utilise l'équation (3) de Robinson, Foster \& Forbes (1969).

\section{Discussion}

Les quantités moyennes de matière sèche ingérée $\left(83 \pm 14 \mathrm{~g} / \mathrm{kg} \mathrm{P}^{0,75}\right)$ sont supérieures de 22 p. 100 à celles obtenues précédemment en allaitement artificiel sur des agneaux de même génotype (68 $\mathrm{g} / \mathrm{kg} \mathrm{P}^{0,75}$, VilletTe \& Theriez, 1981). Ces différences peuvent s'expliquer par des problèmes d'adaptation du jeune à l'allaitement artificiel et/ou par une surestimation de la teneur en M.S. du lait. Les valeurs de l'indice de consommation ne sont cependant que légèrement inférieures à celles obtenues par d'autres auteurs utilisant les méthodes de pesée avant et après tétée sur des agneaux plus âgés (Ricordeau \& Boccard, 1961 ; Peart, 1967).

Les résultats confirment tout au moins pour la première semaine de vie l'influence du poids à la naissance sur les consommations et la croissance qui avait été observée sur des agneaux en allaitement artificiel pendant 6 semaines (VILletTe \& TherIEz, 1981). Dans cette expérience, les diminutions du poids à la naissance sont en partie associées à une augmentation de la taille de la portée mise bas et allaitée. Cette augmentation s'accompagne en début de lactation d'un accroissement de la production laitière (Peart, 1972, 1975 ; Doney, Peart \& SMith, 1981), liée à la demande supérieure effectuée par les agneaux et à l'augmentation du potentiel laitier des mères qui pourrait survenir après une gestation multiple (PEART, 1972). D'après nos résultats, c'est la demande des agneaux qui détermine le niveau de production laitière en première semaine de vie, que ce soit en allaitement simple ou double. Ces résultats diffèrent de ceux obtenus plus tardivement où c'est le potentiel des mères qui détermine, dans certains génotypes, les quantités bues par les agneaux de portée double (Doney, Peart \& Smith, 1981).

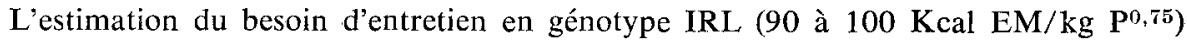
est voisine des valeurs proposées par l'I.N.R.A. (1978) (100 Kcal EM/kg Po,75) et l'A.R.C. (1981) $\left(110 \mathrm{kcal} \mathrm{EM} / \mathrm{kg} \mathbf{P}^{0,75}\right)$. Les valeurs supérieures pour le génotype IDF (140 à $156 \mathrm{kcal}$ EM/P,075) correspondent aux données obtenues en chambre respiratoire par Vermorel (données non publiées) pour des agneaux de même race âgés de 24 heures. Ces différences de besoin d'entretien selon la race doivent refléter des différences de températures ambiantes, mais plus vraisemblablement des différences de toison qui, courte et bouclée en génotype IDF, présente un mauvais pouvoir isolant pendant les premiers jours de vie (Dussuel, KobIlinski \& Berbigier, 1980). Les estimations du kpf sont inférieures à celles calculées par WALKER \& NorToN (1971) $(0,71)$ sur agneaux et par Vermorel et al. $(1974)(0,69)$ sur veaux. Cette sousévaluation ne peut s'expliquer que par une sous-estimation de la teneur en énergie du gain $(2200 \mathrm{Kcal} / \mathrm{kg})$ ou une surestimation du rendement EM/ED $(0,96)$. En effet, pour obtenir une valeur du kpf de 0,68 à partir des droites de régression (tabl. 5), les estimations respectives seraient de $3320 \mathrm{Kcal} / \mathrm{kg}$ pour la valeur énergétique du 
gain ou de 0,63 pour le rapport $E M / E D$, valeurs très éloignées de celles disponibles dans la bibliographie. Ces différences peuvent s'expliquer d'une part par l'incertitude de la mesure du kpf qui demande de nombreuses estimations et d'autre part par une richesse en protéines du gain plus élevée en $1^{\text {re }}$ semaine de vie que pour l'ensemble de la période lactée.

En conclusion, ces premiers résultats, concordants avec ceux obtenus par d'autres méthodes de mesure de production laitière, permettent d'envisager l'utilisation de la technique de dilution d'un marqueur de l'eau pour déterminer les quantités de lait ingéré en début de vie postnatale.

Accepté pour publication en juillet 1983.

\section{Summary}

Milk intake in lambs suckled by their dams during the first week of life

Using an isotope dilution technique the milk intake of 67 one-week old lambs of four breeds Limousin (L), Romanov $\times$ Limousin (RL), Ile-de-France (IDF) and IDF $\times$ RL (IRL) was measured. On a metabolic weight basis $\left(\mathrm{W}^{0.75}\right)$, intakes were similar within breeds or for the number of lambs suckled. They were also independant of birth weigth. With twin suckling, the milk production during the first week depended only on the suckling intensity and increased with litter weight.

The maintenance requirements estimated by regression of live weight gain $(\mathrm{kg} / \mathrm{kg}$ $\left.\mathrm{W}^{0.75}\right)$ on milk intake $\left(\mathrm{kg} / \mathrm{kg} \mathrm{W^{0.75 }}\right)$ were greater in IDF lambs $(140$ to $156 \mathrm{kcal} \mathrm{ME} / \mathrm{kg}$ $\mathrm{W}^{0.75}$ ) than in IRL lambs (90 to $\left.100 \mathrm{kcal} \mathrm{ME} / \mathrm{kg} \mathrm{\textrm {W } ^ { 0 . 7 5 }}\right)$. The values for $\mathrm{kpf}$ were similar within breeds $(0.43$ to 0.51$)$ but smaller than those reported by other authors.

\section{Références bibliographiques}

Agricultural Research Council, 1981. In : The nutrient requirements of ruminant livestock. Commonwealth Agricultural Bureaux, 351 p.

Doney J.N., Peart J.N., SMith W.F., 1981. The effect of interaction of ewe and lamb genotype on milk production of ewes and on growth of lambs to weaning. Anim. Prod., 33, $137-142$.

Dove H., Freer M., 1979. The accuracy of tritiated water turnover rate as an estimate of milk intake in lambs. Aust. J. agric. Res., 30, 725-739.

Dussuel A., Kobilinsky A., Berbigier P., 1980. Régulation thermique de l'agneau nouveauné : analyse de quelques facteurs influençant l'évolution de la température interne et cutanée de l'agneau nouveau-né. Ann. Zootech., 29, 365-376.

EwBank R., 1967. Nursing and suckling behaviour amongst Clun Forest ewes and lambs. Anim. Behav., 15, 251-258.

Gardner R.W., Hogue D.E., Bensadoun A., 1964. The effects of energy intake and number of lambs suckled on milk yield composition and energetic efficiency of lactating ewes. J. anim. Sci., 23, 935-942.

Houssin Y., Davicco M.J., 1979. Influence of birth weight on the digestibility of a milkreplacer in new-born lambs. Ann. Rech. vet., 10, 419.421.

Institut National de la Recherche Agronomique, 1978. In : L'alimentation des ruminants. Ed. I.N.R.A. Publication, 78000 Versailles, 598 p. 
PEART J.N., 1967. The effect of different levels of nutrition during late pregnancy on the subsequent production of Blackface ewes and on the growth of their lambs. J. agric. Sci., Camb., 68, 365-371.

PEART J.N., 1972. The yield and composition of the milk of Finnish Landrace $\times$ Blackface ewes. Ewes land lambs maintained indoors. J. agric. Sci., Camb., 79, 303-313.

PeArT J.N., 1975. The yield and composition of the milk of Finnish Landrace $\times$ Blackface ewes. II - Ewes and lambs grazed on pastures. J. agric. Sci., Camb., 85, 315-324.

Perrin D.R., 1958. The chemical composition of the colostrum and milk of the ewe. J. Dairy. Sci., 25, 70-74.

Ricordeau G., Boccard R., 1961. Relations entre la quantité de lait consommé par les agneaux et leur croissance. Ann. Zootech., 10, 113-125.

Robinson J.J., Foster W.H., Forbes T.J., 1969. The estimation of the milk yield of an ewe from bodyweight data of the suckling lamb. J. agric. Sci. Camb., 72, 103-107.

Sans Arias R., OVejero F.J., Zorita Y.E., 1973. Evolucion de la composicion quimica del colostro de oveja. Ann. Fac. vet. Leon., 19, 273-279.

Tissier M., Robelin J., Purroy A., Geay Y., 1978. Extraction et dosage automatique rapide de l'eau lourde dans les lipides biologiques. Ann. Biol. anim. Biochim. Biophys., 18, $1223-1228$.

Vermorel M., Bouvier J.C., Thivend P., Toullec R., 1974. Utilisation énergétique des aliments d'allaitement par le veau préruminant à l'engrais à différents poids. In : Energy metabolism of farm animals. $6^{\mathrm{e}}$ Symposium, Hohenheim, 1973, 307 p., pp. $143-146$.

Villette Y., Theriez M., 1981. Influence du poids à là naissance sur les performances d'agneaux de boucherie. I - Niveau d'ingestion et croissance. Ann. Zootech., 30, 151.158.

Walker D.M., Norton B.W., 1971. The utilization of the metabolizable energy of diets of different protein content by the milk-fed lamb. J. agric. Sci., Camb., 77, 363-369. 